\title{
Listeria Meningoencephalitis and Hydrocephalus Complicating Alemtuzumab Therapy for Multiple Sclerosis - The First Reported Case in North America
}

\author{
Brian Simms ${ }^{1}$, Bakri Kulla ${ }^{1}$, Jessica Sheehan ${ }^{2}$ and Patrick Haggerty ${ }^{2 *}$ \\ ${ }^{1}$ Specialist in infectious disease, Eastern Virginia Medical School, Norfolk, Virginia, US State
}

${ }^{2}$ Department of Internal Medicine, Division of Infectious Disease, Eastern Virginia Medical School, Norfolk, Virginia, US State

Received: December 22, 2017; Accepted: February 01, 2018; Published: March 19, 2018

*Corresponding author: Patrick Haggerty, Department of Internal Medicine, Division of Infectious Disease, Eastern Virginia Medical School, Norfolk, Virginia, USA, Tel: 757-446-8910; Fax: 757-446-7922; E-mail: HaggerPG@EVMS.edu

\begin{abstract}
Listeria monocytogenes is an opportunistic pathogen known to cause septicemia, meningitis, and rhombencephalitis. Immunosuppression is considered therapeutic when Biologic Response Modifiers (BRMs) are used in the treatment of hematological malignancies, multiple sclerosis and other autoimmune disorders. This report describes a patient receiving one such biological agent, alemtuzumab, for the treatment of multiple sclerosis and the subsequent development of Listerial meningitis complicated by hydrocephalus. This is the first alemtuzumab-associated case of Listeria monocytogenes infection reported in North America and will hopefully spread awareness of this serious but treatable complication.

Abbreviations: bpm: Beats per Minute; CNS: Central Nervous System; CD: Cluster of Differentiation; CT: Computed Tomography; CMV: Cytomegalovirus; L. Monocytogenes: Listeria monocytogenes; MEP-PCR: Meningitis/Encephalitis Panel by Polymerase chain reaction; VP: Ventriculoperitoneal Shunt; WHO: World Health Organization
\end{abstract}

\section{Introduction}

Listeria monocytogenes has been known to cause a wide range of infections from gastroenteritis to meningoencephalitis. $L$. monocytogenes is thought to be responsible for approximately $4 \%$ of all cases of bacterial meningitis [1]. These cases are typically seen in infants and the elderly, the immunocompromised and pregnant women who have consumed soft cheeses, unpasteurized dairy products, or deli meats [2]. The incidence of listeriosis is thought to be declining due to the rise in consumption of prepackaged foods which are less likely to be contaminated with the bacteria [3].

While the incidence of listeriosis may be declining, the number of individuals taking biologic response modifiers is on the rise. Since these biologic, immunosuppressive are now commonly prescribed, one must have a high level of clinical suspicion for diagnosing opportunistic infections [4]. One such biologic agent is alemtuzumab, a humanized monoclonal antibody that targets the CD52 surface marker expressed on B and T lymphocytes and dendritic cells [5]. Alemtuzumab was approved by the FDA in 2014 for the treatment of relapsing Multiple Sclerosis (MS) and was found to be more effective for treating relapsing-remitting MS than interferon beta-1a. Unfortunately, alemtuzumab has also been associated with a greater number of infections [6]. The WHO has reported 21-cases of listeria infection in patients taking alemtuzumab, 16 of which occurred in patients being treated for multiple sclerosis [7]. Here we report a case of Listeria meningoencephalitis in a MS patient after her fourth infusion of alemtuzumab, the first documented case in the United States.

\section{Case Presentation}

The patient is a thirty-five year old African American female who was diagnosed with multiple sclerosis (MS) in 2007; 3 years after her lower extremity paresthesias first began. She was treated with interferon $\beta-1 \alpha$ for six month, interferon $\beta-1 \beta$ for three months, and glatiramer acetate for 6 months; all of these required termination either due to drug reactions or to the development of new lesions seen on MRI. During these trials, her MS had progressed significantly and she was no longer able to ambulate without the assistance of a cane which she would require throughout the remainder of her diagnosis. She was treated with Natalizumab from 2008 through 2011, but this was discontinued when she tested positive for John Cunningham virus. Subsequently she was started back on Glatamir acetate. In 2013 she was switched to Dimethyl fumarate as an MRI obtained at that time showed the development of multiple new MS lesions and remained on this for over a year. During this time, she developed worsening ataxia and three new enhancing lesions on the MRI of head, and several MS flares requiring hospitalization and treatment with high-dose intravenous steroids.

Fingolimod was started in 2015 and was continued until June of 2017 when she began experiencing urinary incontinence and 
worsening short-term memory. Given her functional decline and inability to perform activities of daily living, her physician decided to try alemtuzumab. The first four infusions of her induction cycle were tolerated without difficulty but, prior to receiving her fifth/ last infusion, she complained of mild transient headache but was more concerned about newly developed leg tremors. She was noted to have significantly worsened bilateral leg weakness on physical exam, which prompted a referral to the Emergency Department (ED) for further evaluation. She checked into the ED but left without being seen to attend to family matters and was not again evaluated until one week later. Upon arrival to the clinic to receive her rescheduled fifth infusion of alemtuzumab, she reported several days of intermittent, transient dysarthria and worsening of her chronic leg weakness.

On examination, the patient was found to be dysarthric with significant bilateral leg flaccid paralysis. She was sent to the ED where it was noted that she was tachycardic at 120 Beats per Minute (bpm) with a low-grade fever of $37.6^{\circ} \mathrm{C}$ in the setting of leukocytosis, an elevated lactate, and a Urinalysis (UA) showing pyuria. She was admitted to the hospital and started on empiric IV ceftriaxone. Her dysarthria \& leg weakness significantly improved with IV Solumedrol; however, she remained febrile with temperatures up to $40^{\circ} \mathrm{C}$. Urine cultures showed over $100 \mathrm{k}$ colonies of polymicrobial growth 72 hours into admission while routine blood cultures obtained at that same time showed no growth. Infectious Disease was consulted for persistent fevers and, in the setting of a newly developed transaminitis, recommended empirical Gancyclovir for CMV coverage.

Repeat blood cultures several days into admission showed gram positive rods with a preliminary read of Diphtheroids and as a likely contaminant. These cultures were identified as Listeria Monocytogenes 24 hours later and her antimicrobials were switched to Ampicillin/Gentamicin for disseminated infection. Later that day, she was found unresponsive with fixed/ dilated pupils and immediately intubated. Non-contrast head CT showed significant hydrocephalus that required the placement of an emergent bedside ventriculostomy. CSF sampled from her ventriculostomy showed the following: RBCs 42 cells $/ \mathrm{mm} 3$, WBC 120 cells $/ \mathrm{mm} 3$ with $87 \%$ neutrophils and $8 \%$ lymphocytes, protein of $47 \mathrm{mg} / \mathrm{dL}$, and glucose of $35 \mathrm{mg} / \mathrm{dL}$. Meningitis/ Encephalitis Panel by Polymerase chain reaction (MEP-PCR-CSF) returned positive for L. monocytogenes which grew on CSF culture several days later. Gentamicin was discontinued one week later due to a rising creatinine but the patient remained on Ampicillin for the duration of her planned therapy.

Ventriculostomy was later removed when she began to show neurologic improvement, being increasingly alert and able to follow commands. She unfortunately developed altered mental status later in her hospitalization; CT-Head showed worsening hydrocephalus and the patient required emergent VP shunt placement.

Patient has since shown clinical improvement, being able to follow commands and move all her extremities, and was discharged to a skilled nursing facility for continued rehabilitation.

\section{Discussion}

Listeria monocytogenes is a gram positive, facultative anaerobic bacillus that has the unique ability to grow freely at refrigerator temperatures $\left(4-10^{\circ} \mathrm{C}\right)$; this predilection towards colder temperatures isolates it from other bacteria and allows for recovery rates of $15-70 \%$ from raw vegetables, milk, cheese, and frozen/processed meats [8]. Given its prevalence, the ingestion of Listeria is assumed to be a common nonpathogenic occurrence. Invasive disease however is a rare occurrence with an incidence of 2-3 cases per million populations in the United State [9] $(0.1 \%$ of all food borne infections) [10]. Listeria is a known cause of meningitis, encephalitis, and brain abscesses particularly in patients who are immunocompromised. Interestingly, Listerial associated CNS infections have incidence rate of $47 \%$ of all documented cases, and the mortality rate due to CNS involvement is up to $30 \%$ regardless of appropriate treatment [10].

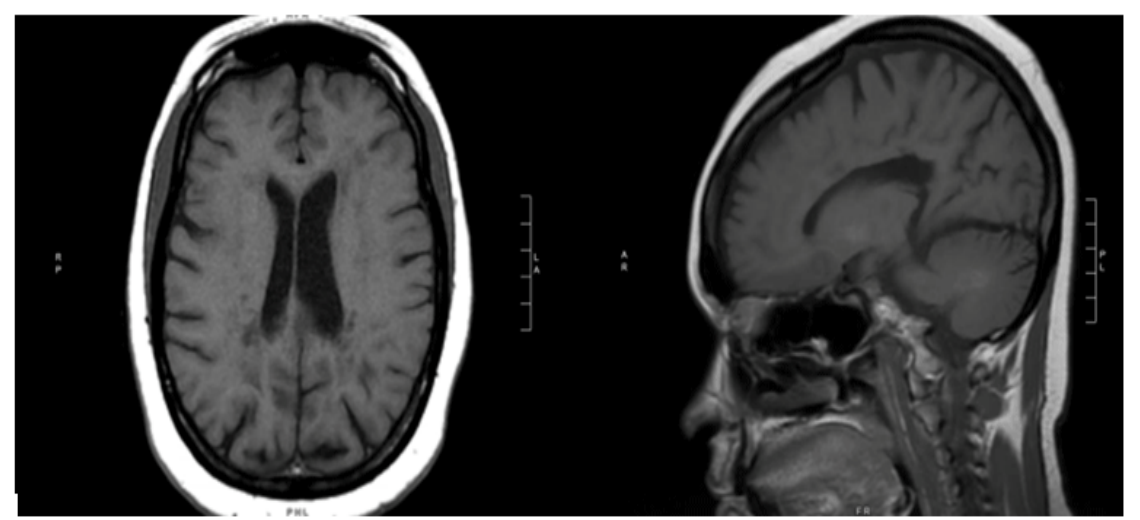

Figure 1:T1 MRI four months prior to admission. No hydrocephalus noted. MRI read: Extensive changes of multiple sclerosis both supratentorial and infratentorial with extensive involvement of the atrophic corpus callosum. New areas of demyelination involving anterior cerebellum and left middle cerebellar peduncle. Continued extensive associated T1 hypointense lesions. No other new acute intracranial findings. 
While the pathogenicity of L. monocytogenes is not completely understood, it is known to be able to translocate through the gastrointestinal tract and travel to the liver where it begins to replicate. L. monocytogenes is an obligate intracellular organism that moves hepatocyte-to-hepatocyte via an actin-based intercellular spread, thereby allowing it to avoid contact with antibodies. Thus, immunity against the bacteria is primarily dependent on CD8+ T cells to detect listerial epitopes and kill infected hepatocytes $[8,11]$. The host's humoral response is therefore vital for the clearance of this organism. In particular, Listeria is known to infect pregnant patients who experience a normophysiologic impairment of their cell-mediated immunity. Pregnancy accounts for 25-33\% of all reported Listeriosis cases. Other risk groups include infants younger than 1 month, the elderly, and patients with hematologic malignancies and/or immunodeficiency's.

Our patient's immunosuppression was due to both Alemtuzumab infusions and corticosteroid use, both of which put her at an increased risk of infection. Alemtuzumab has been shown to be efficacious in the treatment of various hematological malignancies and relapsing-remitting multiple sclerosis via rapid depletion of both B and T lymphocytes. Therapy regimens typically include a "first cycle" of 5 consecutive daily infusions followed by another 3 infusions 12 months later. It is currently recommended that MS patients receive prophylactic treatment only for herpes simplex virus [12]. Patients have shown an increased likelihood of latent CMV reactivation which may warrant close monitoring or even prophylactic use of valganciclovir in lieu of acyclovir [13].

As with other biological agents, alemtuzumab is able to cause infusion-related reactions that can be difficult to differentiate from a newly developing infectious process. The severity of these reactions has decreased significantly since the introduction of corticosteroid into the regimen but can still cause fevers, nausea, vomiting, dyspnea, cough, and diarrhea [14]. It should be noted that the use of steroids has been associated with prolonged colonization by L. monocytogenes after exposure to contaminated foods [15].

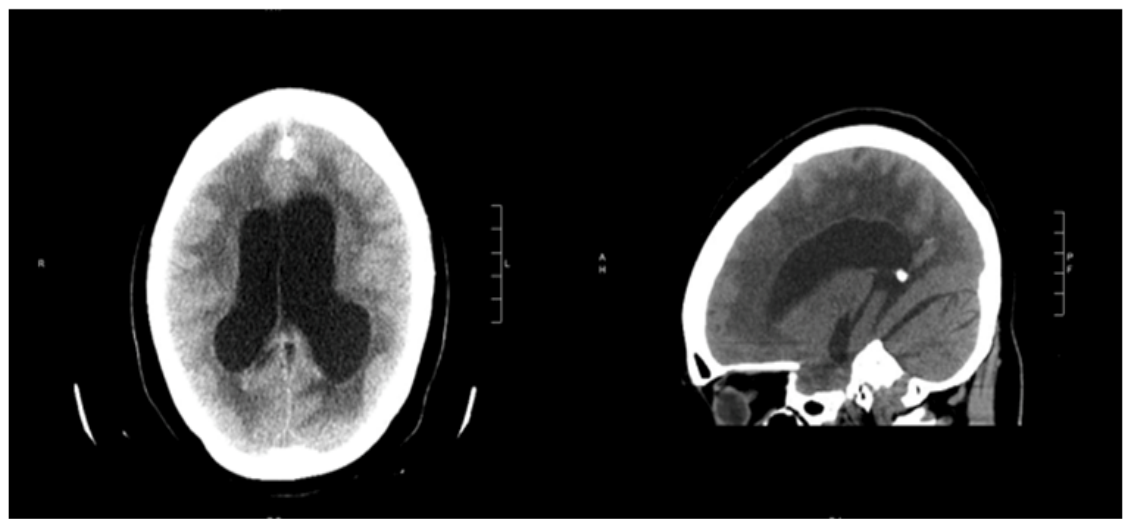

Figure 2: Non-contrast head CT prior to placement of emergent VP shunt. CT read: Interval development of severe hydrocephalus. Cortical sulci of the cerebral hemispheres are effaced. There is dilatation of the lateral ventricles and third ventricle. Suspect obstruction at the cerebral aqueduct level.

As reported by Holmøy et al, Alemtuzumab infusions have been known to rapidly increase both pro-inflammatory cytokines and acute phase proteins including C-reactive protein levels that can mimic early-onset sepsis7. The transient increase in inflammatory markers seen after treatment with Alemtuzumab can be explained by the drug's mechanism of action. The binding of Alemtuzumab to CD52 initiates the classical pathway of complement activation which leads to cellular lysis [14]. Both this and the resulting antibody-dependent cellular-mediated cytotoxicity pathways result in the rapid depletion of these targeted cells and, when combined with these inflammatory changes, leaves patients susceptible to opportunistic infection even early in their treatment course. Given that our patient's symptoms developed prior to completing her first treatment cycle of 5 daily infusions, it is likely that she was already colonized with L. monocytogenes when the rapid depletion of her lymphocytes allowed for dissemination of the bacteria. While the majority of reported cases of listeriosis in association with Alemtuzumab have occurred within days of the last infusion7, there has been a report of a 36-year-old female receiving two annual cycles of Alemtuzumab that presented with symptoms 13 days after her last infusion [16].

As reported to VigiBase, most alemtuzumab-associated Listerial infections present with signs of meningeal irritation with headache being the most common [17] symptom usually preceded by a prodrome of fevers, vomiting, and nuchal rigidity [11]. One unique aspect of our patient's presentation was her slurred speech and worsening chronic leg paralysis one week after receiving infusions as most documented cases of alemtuzumabassociated listeriosis present with signs of meningeal irritation [18]. Another interesting aspect of the case was that her meningitis was complicated by symptomatic hydrocephalus requiring several emergent decompressions. Hydrocephalus is a known complication of Listerial infections and occurs in approximately $14 \%$ of cases [18] but to our knowledge has not been reported in this specific patient population. The diagnosis of 
listeriosis is complicated by the fact that CSF and blood cultures are only positive in $83 \%$ and $64 \%$ of cases, respectively [18].

CSF Gram stain is positive in 33\% of patients with Listerial meningitis (other study estimated it at $6 \%$ only [18] and because of this, PCR-analysis is often necessary. Proper diagnosis was likely delayed in our patient because her initial blood cultures were negative and repeat cultures were initially thought to be contaminated by Diphtheroids.
To our knowledge, the patient described in this report is the 1st documented case of Listerial meningitis complicating alemtuzumab therapy in North America. As this complication is exceedingly rare with over 11,500 patients receiving this therapy and only 23 reported cases of listeriosis, no current recommendations exist for the preemptive treatment of listerial colonization.

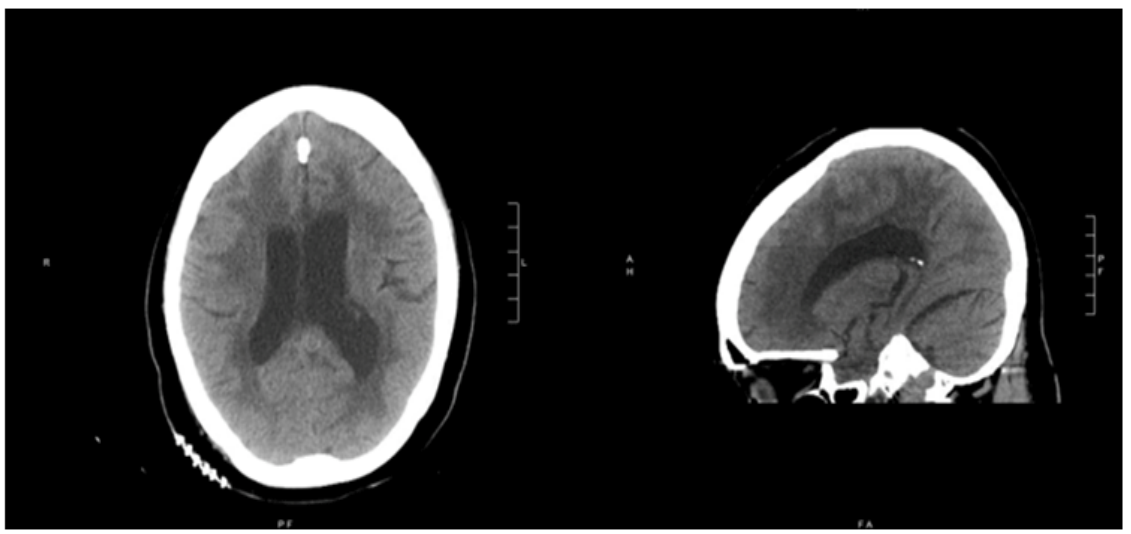

Figure 3: Non-contrast head CT 6 days post VP shunt placement. CT read: Improving hydrocephalus, moderate remains. Improved cerebral edema. Persistent diffuse nonspecific white matter abnormalities.

Lemtrada's Summary of Product Characteristics recommends currently that patients avoid ingestion of risky foods for one month after having stopped infusions. Given that intestinal colonization with Listeria monocytogenes is not uncommon, it would be ideal for patients to stop eating any potentially contaminated food several months prior to the start of therapy. Many of the articles referenced by our team support this recommendation and advise patients take small measures to avoid infection by washing fruits and vegetables, thoroughly cooking meat, and avoiding soft cheeses, dairy products, and deli meats during the months surrounding their infusions.

\section{Conclusion}

Listeria is an important and potentially lethal pathogen that can cause serious infections and complications in immune compromised patients. The increased use of biologics in the treatment of many immunological diseases may also increase the incidence of this disease as many therapies limit the patients ability to mount an appropriate cell mediated immune response.

This case report highlights the first documented case of $L$. monocytogenes as a cause of meningitis in patients receiving alemtuzumab in North America that was also complicated by hydrocephalus. The atypical presentation of our patient serves to stress the high degree of clinical suspicion physicians should maintain when working with this particular patient population. Current recommendations for patients per alemtuzumab's SPC are that patients should receive prophylactic treatment only for herpes simplex virus. Practitioners should however have a high index of suspicion for CMV reactivation and consider the use of valganciclovir if necessary. In addition, based on the references reviewed for this case report, most advice that patients avoid high risk foods in the months surrounding therapy and those clinicians consider preemptive treatment of L. monocytogenes if patients develop neurological symptoms.

\section{References}

1. Thigpen MC, Whitney CG, Messonnier NE, Zell ER, Lynfield R, Hadler JL, et al. Bacterial meningitis in the United States, 1998-2007. N Engl J Med. 2011;364(21):2016-2025. DOI: 10.1056/NEJMoa1005384

2. Schuchat A, Deaver KA, Wenger JD, Plikaytis BD, Mascola L, Pinner RW, et al. Role of foods in sporadic listeriosis. I. Case-control study of dietary risk factors. The Listeria Study Group. JAMA. 1992;267(15):20412045.

3. Voetsch AC, Angulo FJ, Jones TF, Moore MR, Nadon C, McCarthy P, et al. Reduction in the incidence of invasive listeriosis in foodborne diseases active surveillance network sites, 1996-2003. Clin Infect Dis. 2007;44(4):513-520. DOI: 10.1086/511006

4. Singh JA, Wells GA, Christensen R, Tanjong Ghogomu E, Maxwell L, Macdonald JK, et al. Adverse effects of biologics: a network metaanalysis and Cochrane overview. Cochrane Database Syst Rev. 2011(2):CD008794. DOI: 10.1002/14651858.CD008794.pub2

5. Havrdova E, Horakova D, Kovarova I. Alemtuzumab in the treatment of multiple sclerosis: key clinical trial results and considerations for use. Ther Adv Neurol Disord. 2015;8(1):31-45. DOI: $10.1177 / 1756285614563522$

6. Coles AJ, Compston DA, Selmaj KW, Lake SL, Moran S, Margolin DH, et al. Alemtuzumab vs. interferon beta-1a in early multiple sclerosis. $\mathrm{N}$ Engl J Med. 2008;359(17):1786-1801. DOI: 10.1056/NEJMoa0802670 
7. Holmøy T, von der Lippe H, Leegaard TM. Listeria monocytogenes infection associated with alemtuzumab - - a case for better preventive strategies. BMC Neurol. 2017;17(1):65. DOI: 10.1186/s12883-0170848-8

8. Mandell G, Bennett J, Dolin R. Mandell, Douglas, and Bennett's Principles and Practice of Infectious Diseases. 7th Edition ed: Churchill Livingston; 2009.

9. Vital signs: Listeria illnesses, deaths, and outbreaks--United States, 2009-2011. MMWR Morb Mortal Wkly Rep. (CDC) CfDCaP. 2013;62(22):448-452.

10. Rau D, Lang M, Harth A, Naumann M, Weber F, Tumani H, et al Listeria Meningitis Complicating Alemtuzumab Treatment in Multiple Sclerosis--Report of Two Cases. Int J Mol Sci. 2015;16(7):1466914676. DOI: $10.3390 /$ ijms160714669

11.Vázquez-Boland JA, Kuhn M, Berche P, Chakraborty T, Bernal GD, Goebel W, et al. Listeria pathogenesis and molecular virulence determinants. Clin Microbiol Rev. 2001;14(3):584-640. DOI: 10.1128/ CMR.14.3.584-640.2001

12. Koo S, Marty FM, Baden LR. Infectious complications associated with immunomodulating biologic agents. Hematol Oncol Clin North Am. 2011;25(1):117-138. DOI: 10.1016/j.hoc.2010.11.009
13. O’Brien S, Ravandi F, Riehl T, Wierda W, Huang X, Tarrand J, et al. Valganciclovir prevents cytomegalovirus reactivation in patients receiving alemtuzumab-based therapy. Blood. 2008;111(4):18161819. DOI: 10.1182/blood-2007-03-080010

14. Laros-van Gorkom BA, Huisman CA, Wijermans PW, Schipperus MR. Experience with alemtuzumab in treatment of chronic lymphocytic leukaemia in the Netherlands. Neth J Med. 2007;65(9):333-338.

15. Prats N, López S, Domingo M, Briones V, García JA, Domínguez Let al. Prolonged persistence of Listeria monocytogenes after intragastric infection in corticosteroid-treated mice. Vet Microbiol. 1997;58(1):7985.

16. Wray S. On behalf of the CAMMS223 Study Group. Descriptive analysis of infectious adverse events in alemtuzumab-treated multiple sclerosis patients. In Proceedings of the 25th Congress of the European Committee for Treatment and Research in Multiple Sclerosis, Düsseldorf, Germany, 9-12 September 2009; p. S247. In.

17.VigiBase, the World Health Organization (WHO) international database of suspected adverse drug reactions. Accessed November $30,2017$.

18. Pelegrín I, Moragas M, Suárez C, Ribera A, Verdaguer R, MartínezYelamos $\mathrm{S}$, et al. Listeria monocytogenes meningoencephalitis in adults: analysis of factors related to unfavourable outcome. Infection. 2014;42(5):817-827. DOI: 10.1007/s15010-014-0636-y 